\title{
On two recurrence problems
}

by

Michael Boshernitzan (Houston, TX) and Eli Glasner (Tel Aviv)

\begin{abstract}
We review some aspects of recurrence in topological dynamics and focus on two open problems. The first is an old one concerning the relation between Poincaré and Birkhoff recurrence; the second, due to the first author, is about moving recurrence. We provide a partial answer to a topological version of the moving recurrence problem.
\end{abstract}

Introduction. Poincaré's recurrence theorem is the first and most basic theorem of ergodic theory. It asserts that given a measure preserving (invertible) dynamical system $(X, \mu, X, T)$ and $A \in X$ with $\mu(A)>0$, the set $N(A, A)=\left\{n \in \mathbb{Z}: \mu\left(T^{n} A \cap A\right)>0\right\}$ meets every set of the form $(L-L) \backslash\{0\}=\{n-m: n, m \in L, n \neq m\}$ with infinite $L \subset \mathbb{Z}$. The proof of this surprising fact is straightforward: The sets $T^{n} A, n \in L$, having the same (positive) measure, cannot be all disjoint $(\bmod \mu)$. If $\mu\left(T^{n} A \cap T^{m} A\right)>0$ then $\mu\left(T^{n-m} A \cap A\right)>0$, hence $n-m \in N(A, A)$.

This basic measure-theoretic recurrence theorem has a topological counterpart due to G. D. Birkhoff. If $(X, T)$ is a topological dynamical system ( $X$ is a compact metric space and $T: X \rightarrow X$ is a homeomorphism of $X$ onto itself), then there is a recurrent point in $X$, i.e. there is a point $x \in X$ such that for every $\epsilon>0$ there is some $n \geq 1$ with $d\left(x, T^{n} x\right)<\epsilon$. A purely topological proof of this theorem (i.e. one which does not use the fact that such a system always admits an invariant probability measure and then applies Poincaré's theorem) follows from the fact that minimal subsystems always exist. One first applies Zorn's lemma to show that every compact topological system admits a minimal subset and then uses the characterization of a point whose orbit closure is minimal as a uniformly recurrent point (see Lemma 4.3 below).

Poincaré's and Birkhoff's recurrence theorems obtained more recently a deep and far reaching generalization in the form of Furstenberg's multiple

2010 Mathematics Subject Classification: Primary 37B20, 54H20.

Key words and phrases: recurrence, moving recurrence, syndetic sets, Birkhoff sets, Poincaré sets, Bohr neighborhoods of zero. 
recurrence theorem, from which Furstenberg was able to deduce the famous theorem of Szemerédi: a subset $A \subset \mathbb{N}$ of positive upper Banach density contains arbitrarily long arithmetical progressions (see Furstenberg [10]).

In the present work we review some aspects of recurrence in topological dynamics as developed by Gottschalk and Hedlund [17], and Furstenberg [10], including several "folklore" theorems, and then focus on two particular open problems. The first is an old one concerning the relation between Poincaré and Birkhoff recurrence (see Problems $(\mathrm{A}),\left(\mathrm{A}^{\prime}\right)$ and $\left(\mathrm{A}^{\prime \prime}\right)$, or 2.5, 4.9 and 4.11, respectively), and the second, due to the first author, is about "moving recurrence" (see Section 6). While the original "moving recurrence" problem remains open, we provide here a partial answer to a topological version of the problem (Theorem 6.4). The paper also contains other new results on topological recurrence. In particular, in Section 5 we introduce the notion of $r$-Birkhoff sets (approximating that of Birkhoff sets) and present some preliminary results concerning these sets. Finally, in Section 7 we show that "absolute moving recurrence" is equivalent to uniform rigidity.

For related works see [10], [26], [2], [12], [28], [3], [19], [14] and [4]. A comprehensive review by Frantzikinakis and McCutcheon on the subject of recurrence in dynamics is forthcoming [9].

\section{A reminder of some preliminary definitions and basic results.}

In this work a dynamical system is a pair $(X, T)$, where $X$ is a compact space and $T: X \rightarrow X$ is a homeomorphism of $X$ onto itself. For subsets $A$ and $B$ of $X$, we let

$$
N(A, B)=\left\{n \in \mathbb{Z}: T^{n} A \cap B \neq \emptyset\right\} .
$$

When $A=\{x\}$ is a singleton we write $N(A, B)=N(x, B)$, thus

$$
N(x, B)=\left\{n \in \mathbb{Z}: T^{n} x \in B\right\} .
$$

For a point $x \in X$ we write $\mathcal{O}_{T}(x)=\left\{T^{n} x: n \in \mathbb{Z}\right\}$ for the orbit of $x$ and $\overline{\mathcal{O}}_{T}(x)$ for the closure of $\mathcal{O}_{T}(x)$. We say that the system $(X, T)$ is point transitive if there is a point $x \in X$ with $\mathcal{O}_{T}(x)$ dense. Such a point is called transitive. We say that the system $(X, T)$ is topologically transitive (or just transitive) if the set $N(U, V)$ is nonempty for every pair $U$ and $V$ of nonempty open subsets of $X$. Clearly, point transitivity implies topological transitivity and using Baire's category theorem one can show that, conversely, in a topologically transitive metric system the set $X_{\operatorname{tr}}$ of points whose orbit is dense forms a dense $G_{\delta}$ subset of $X$. A point $x \in X$ is a recurrent point if the set $N(x, U) \backslash\{0\}$ is nonempty for every neighborhood $U$ of $X$. A dynamical system is called minimal if every point is transitive.

A dynamical system $(X, T)$ is equicontinuous if the collection of maps $\left\{T^{n}: n \in \mathbb{Z}\right\}$ is equicontinuous. A minimal equicontinuous system is called a Kronecker system. We have the following classical theorem: 


\subsection{THEOREM.}

(1) A metrizable dynamical system $(X, T)$ is equicontinuous if and only if there is a compatible metric on $X$ with respect to which $T$ is an isometry.

(2) A dynamical system is Kronecker if and only if it is isomorphic to a system of the form $\left(G, R_{a}\right)$, where $G$ is a compact monothetic topological group, $a \in G$ is a topological generator (meaning that the cyclic subgroup $\left\{a^{n}: n \in \mathbb{Z}\right\}$ is dense in $G$ ), and the transformation $R_{a}$ is defined by $R_{a} g=g a$.

There exists a largest monothetic compact topological group $b \mathbb{Z}$ called the Bohr compactification of the integers. If we let $\phi: \mathbb{Z} \rightarrow b \mathbb{Z}$ be the canonical map $\phi: \mathbb{Z} \rightarrow b \mathbb{Z}$, then $a=\phi(1)$ is a topological generator of the group $b \mathbb{Z}$ and one can associate to $b \mathbb{Z}$ a dynamical system $\left(b \mathbb{Z}, R_{a}\right)$ as above. This system is minimal and equicontinuous, but nonmetrizable. It is the largest minimal equicontinuous system in the sense that every other such system is a factor of $\left(b \mathbb{Z}, R_{a}\right)$.

For more information on these basic notions of topological dynamics refer e.g. to Chapter 1 of [13]. In the rest of this work we will assume, unless we explicitly say otherwise, that our dynamical systems are metrizable.

\section{Some families of subsets of $\mathbb{Z}$ and a famous open problem.} In order to avoid some tedious repetitions we introduce the notation $\mathbb{Z}_{*}=$ $\mathbb{Z} \backslash\{0\}$.

2.1. Definition. Let $L \subset \mathbb{Z}_{*}$.

(1) $L$ is a Poincaré set if whenever $(X, X, \mu, T)$ is a probability preserving system and $A \subset X$ is a positive set (i.e. $A \in \mathcal{X}$ and $\mu(A)>0$ ), then $N(A, A) \cap L \neq \emptyset$. Let Po denote the collection of Poincaré subsets of $\mathbb{Z}_{*}$.

(2) It is a Birkhoff set (or a set of topological recurrence) if whenever $(X, T)$ is a minimal dynamical system and $U \subset X$ a nonempty open set, then $N(U, U) \cap L \neq \emptyset$. Let Bir denote the collection of Birkhoff subsets of $\mathbb{Z}_{*}$.

(3) It is a Bohr set if whenever $(X, T)$ is a Kronecker dynamical system and $V \subset X$ a nonempty open set, then $N(V, V) \cap L \neq \emptyset$. Let Bo denote the collection of Bohr subsets of $\mathbb{Z}_{*}$.

\subsection{Definition.}

(1) A subset $\mathcal{F}$ of the power set $\mathcal{P}$ of $\mathbb{Z}_{*}$ is called a family when it is upwards hereditary. That is, $F_{1} \subset F_{2}$ and $F_{1} \in \mathcal{F}$ imply $F_{2} \in \mathcal{F}$.

(2) If $\mathcal{E}$ is any nonempty subset of $\mathcal{P}$, we let $\mathcal{F}(\mathcal{E})$ be the smallest family containing $\mathcal{E}$. 
(3) If $\mathcal{E}$ is any nonempty subset of $\mathcal{P}$ we let its dual $\mathcal{E}^{*}$ be defined by

$$
\mathcal{E}^{*}=\{F: F \cap E \neq \emptyset \text { for all } E \in \mathcal{E}\} .
$$

It is easy to check that $\mathcal{E}^{*}$ is a family and that $\mathcal{F}(\mathcal{E})^{*}=\mathcal{E}^{*}$. Clearly, $\mathcal{F}_{1} \subset \mathcal{F}_{2} \Rightarrow \mathcal{F}_{2}^{*} \subset \mathcal{F}_{1}^{*}$ and finally for a family $\mathcal{F}=\mathcal{F}^{* *}$.

\subsection{Definition.}

(1) Let $\mathcal{E}_{\text {Po }}$ be the collection of all subsets of $\mathbb{Z}_{*}$ of the form $N(A, A)$, whenever $(X, \mu, T)$ is a probability preserving system and $A \subset X$ is a positive set $(\mu(A)>0)$. We then have $\mathbf{P o}=\mathcal{F}\left(\mathcal{E}_{\mathrm{Po}_{\mathrm{o}}}\right)^{*}=\mathcal{E}_{\mathrm{Po}}^{*}$.

(2) Let $\mathcal{E}_{\text {Bir }}$ be the collection of all subsets of $\mathbb{Z}_{*}$ of the form $N(U, U)$, whenever $(X, T)$ is a minimal system and $U \subset X$ is a nonempty open subset of $X$. We have $\operatorname{Bir}=\mathcal{F}\left(\mathcal{E}_{\text {Bir }}\right)^{*}=\mathcal{E}_{\text {Bir }}^{*}$.

(3) Let $\mathcal{E}_{\mathrm{Bo}}$ be the collection of all subsets of $\mathbb{Z}_{*}$ of the form $N(V, V)$, whenever $(X, T)$ is a Kronecker system and $V \subset X$ is a nonempty open subset of $X$. We have $\mathbf{B o}=\mathcal{F}\left(\mathcal{E}_{\mathrm{Bo}}\right)^{*}=\mathcal{E}_{\mathrm{Bo}}^{*}$.

\subsection{LEMMA.}

$$
\mathcal{E}_{\mathrm{Po}} \supset \mathcal{E}_{\mathrm{Bir}} \supset \mathcal{E}_{\mathrm{Bo}}, \quad \text { whence } \quad \text { Po } \subset \text { Bir } \subset \text { Bo. }
$$

Proof. If $(X, T)$ is a minimal system then the collection $M_{T}(X)$ of Borel probability measures on $X$ is never empty and if $U \subset X$ is open and nonempty, then $\mu(U)>0$ for every $\mu \in M_{T}(X)$. This implies $\mathcal{E}_{\text {Po }} \supset \mathcal{E}_{\text {Bir }}$. The inclusion $\mathcal{E}_{\text {Bir }} \supset \mathcal{E}_{\text {Bo }}$ follows trivially from the definitions. Finally, the last two inclusions follow by duality.

A beautiful result of Kř́ž [21] (see also [23]) shows that $\mathcal{F}\left(\mathcal{E}_{\mathrm{Po}}\right) \supsetneq \mathcal{F}\left(\mathcal{E}_{\mathrm{Bir}}\right)$.

2.5. Problem (A). Is it also true that $\mathcal{F}\left(\mathcal{E}_{\text {Bir }}\right) \supsetneq \mathcal{F}\left(\mathcal{E}_{\text {Bo }}\right)$ ?

2.6. Remark. Since $\mathbf{P o}=\mathcal{F}\left(\mathcal{E}_{\mathrm{Po}}\right)^{*}$ and $\mathbf{B i r}=\mathcal{F}\left(\mathcal{E}_{\mathrm{Bir}}\right)^{*}$, Kř́íz' result is the same as the statement $\mathbf{P o} \subsetneq \mathbf{B i r}$, and Problem (A) is equivalent to the question whether Bir $\subsetneq$ Bo.

Recall that a collection $\mathcal{E}$ of subsets of $\mathbb{Z}$ is divisible [11] (or has the Ramsey property [10]) if whenever $A$ is in $\mathcal{E}$ and $A=C \cup D$, then at least one of the sets $C$ and $D$ is in $\mathcal{E}$.

2.7. Proposition.

(1) The collection $\mathcal{E}_{\text {Bir }}$ forms a filter base; hence $\mathcal{F}\left(\mathcal{E}_{\text {Bir }}\right)$ is a filter.

(2) The family Bir is divisible.

Proof. Let $(X, T)$ and $(Y, T)$ be minimal dynamical systems, and $U \subset X$, $V \subset Y$ nonempty open sets. Let $M_{0} \subset X \times Y$ be a minimal subset of the product system $(X \times Y, T \times T)$. Since clearly the $\mathbb{Z}^{2}$-action defined on $X \times Y$ by the group $\left\{T^{i} \times T^{j}:(i, j) \in \mathbb{Z}^{2}\right\}$ is minimal, there is a pair $(i, j)$ such that 
$\left(T^{i} \times T^{j}\right) M_{0} \cap U \times V \neq \emptyset$. Set $M=\left(T^{i} \times T^{j}\right) M_{0}$ and $W=(U \times V) \cap M$. Then the system $(M, T \times T)$ is minimal, the set $W$ is a nonempty open subset of $M$, and clearly

$$
N(U, U) \cap N(V, V) \supset N(W, W) .
$$

Thus $\mathcal{E}_{\text {Bir }}$ is indeed a filter base. It follows that $\mathcal{F}\left(\mathcal{E}_{\text {Bir }}\right)$, which is defined as the smallest family containing $\mathcal{E}_{\mathrm{Bir}}$, is a filter. We leave it as an exercise to show that the dual family of a filter has the Ramsey property (and vice versa). Therefore $\mathbf{B i r}=\mathcal{F}\left(\mathcal{E}_{\text {Bir }}\right)^{*}$ has the Ramsey property.

The proof of the next statement is straightforward.

2.8. Proposition. The families $\mathbf{P o}$ and $\mathbf{B o}$ are divisible.

\section{Examples}

\subsection{EXAMPLES.}

- For every infinite $L \subset \mathbb{Z}$ the difference set $\{n-m: m, n \in L, n>m\}$ is a Poincaré set. In fact, this statement is just Poincaré's recurrence theorem.

- Let $p(t)$ be a polynomial with real coefficients taking integer values on the integers and such that $p(0)=0$. Then the sequence $\{p(n)\}_{n \geq 1}$ is Poincaré (see [10, Theorem 3.16]). In particular, the sequence $\left\{n^{2}\right\}_{n \geq 1}$ is Poincaré. It is easy to see that the sequence $\left\{n^{2}+1\right\}_{n \geq 1}$ is not Poincaré. (Consider a cyclic permutation of three points and observe that $n^{2}+1$ is not divisible by 3 .)

- Every thick set $L \subset \mathbb{Z}$ is Poincaré [10, p. 74] (see Definition 4.1.2 below). For the reader's convenience let us reproduce one of the proofs given in [10]. Let $(X, X, \mu, T)$ be a measure preserving system and $A \in \mathcal{X}$ with $0<\mu(A)$. If $A$ is not invariant (i.e. $\mu(T A \triangle A)>0$ ) then there exists an $N \geq 1$ such that $\mu\left(\bigcup_{j=0}^{N} T^{j} A\right)>\mu\left(\bigcup_{j=0}^{\infty} T^{j} A\right)-\mu(A)$. Then, for any $M \geq 1$,

$$
\mu\left(\bigcup_{j=M}^{M+N} T^{j} A\right)>\mu\left(\bigcup_{j=0}^{\infty} T^{j} A\right)-\mu(A) .
$$

This implies that $\mu\left(\bigcup_{j=M}^{M+N} T^{j} A \cap A\right)>0$, for otherwise

$$
\mu\left(\bigcup_{j=0}^{\infty} T^{j} A\right) \geq \mu(A)+\mu\left(\bigcup_{j=M}^{M+N} T^{j} A\right)>\mu\left(\bigcup_{j=0}^{\infty} T^{j} A\right) .
$$

Thus each sufficiently long interval of integers includes an $n$ with $\mu\left(T^{n} A \cap A\right)>0$, and this proves that a thick set is Poincaré. Another way to see this is to show that every thick set contains an infinite difference set as in the first example above. 
- Recall that a sequence of integers $\left\{n_{k}\right\}_{k=1}^{\infty}$ is lacunary if $\liminf _{k} n_{k+1} / n_{k}$ $>1$. An elegant argument by Y. Katznelson (see [20] or [28, Theorem 5.3]) shows that a lacunary sequence is never Bohr. In fact, there is a stronger result (answering a question of Erdös in [8]) according to which for any lacunary sequence of integers $\left\{n_{k}\right\}_{k=1}^{\infty}$, there always exists an irrational $\alpha \in \mathbb{R}$ such that $\inf _{k}\left\|n_{k} \alpha\right\|>0$ (see [25], [22]). (We write $\|x\|=\min _{i \in \mathbb{Z}}|x-i|$ for the distance of a real number $x$ from the set of integers.)

- The results in the above example do not extend to slower growing sequences (see [1], [5]). An increasing sequence of integers $\left\{n_{k}\right\}_{k=1}^{\infty}$ is called sublacunary if $\lim _{k} n_{k+1} / n_{k}=1$. There are various results ([1], [5], [6]) which indicate that for a "generic" sublacunary sequence $\left\{n_{k}\right\}_{k=1}^{\infty}$ the $\operatorname{limits}_{\lim _{k}} N^{-1} \sum_{k=1}^{N} \exp \left(2 \pi i n_{k} \alpha\right)$ exist and vanish for all real $\alpha \notin \mathbb{Z}$. Such sequences are known to be Poincaré and, in particular, Bohr. (In the three papers quoted above the term "generic" has various probabilistic meanings.)

\section{A second formulation of the problem}

\subsection{Definition.}

(1) A subset $S \subset \mathbb{Z}$ is called syndetic if there is a positive integer $N$ such that $S+\{0,1, \ldots, N\}=\mathbb{Z}$.

(2) A subset $R \subset \mathbb{Z}$ is called thick (or replete) if for every positive integer $N$ there is an $n \in \mathbb{Z}$ such that $\{n, n+1, n+2, \ldots, n+N\} \subset R$.

(3) A point $x \in X$, where $(X, T)$ is a dynamical system, is uniformly recurrent if $N(x, U)$ is syndetic for every neighborhood $U$ of $x$. (In [17] a uniformly recurrent point is called an almost periodic point.)

The following proposition is an easy exercise.

4.2. Proposition. Let $\mathcal{S}$ and $\mathcal{T}$ denote the families of syndetic and thick sets respectively. Then $\mathcal{S}$ and $\mathcal{T}$ are dual families.

We have the following important lemma (see [17]).

4.3. Lemma. Let $(X, T)$ be a dynamical system and $x_{0} \in X$. Then $\overline{\mathcal{O}}_{T}\left(x_{0}\right)$ is a minimal subset of $X$ if and only if $x_{0}$ is uniformly recurrent.

Proof. Suppose first that $x_{0} \in X$ has a minimal orbit closure $Y=$ $\overline{\mathcal{O}}_{T}\left(x_{0}\right)$. Let $U$ be a neighborhood of $x_{0}$ in $X$. By minimality there is an $N \geq 1$ such that $Y \subset \bigcup_{j=0}^{N} T^{j} U$. Now given $n \in \mathbb{Z}$ there is some $0 \leq j \leq N$ with $T^{n} x_{0} \in T^{j} U$. Thus $T^{n-j} x_{0} \in U$, hence $n-j \in N\left(x_{0}, U\right)$, hence $n=m+j$ for some $m \in N\left(x_{0}, U\right)$.

Conversely, suppose $x_{0}$ is uniformly recurrent. Set $Y=\overline{\mathcal{O}}_{T}\left(x_{0}\right)$ and let $M \subset Y$ be a minimal subset of $Y$. Suppose $M \neq Y$; then $x_{0} \notin M$. Let $U$ 
and $V$ be open subsets of $X$ such that $x_{0} \in U, V \supset M$ and $U \cap V=\emptyset$. Pick some $y_{0} \in M$. Then the whole orbit of $y_{0}$ is contained in $V$ and for every $N \geq 1$ we can find $n_{N}$ with $T^{n_{N}} x_{0}$ sufficiently close to $y_{0}$ to ensure that $T^{n_{N}} x_{0}, T^{n_{N}+1} x_{0}, \ldots, T^{n_{N}+N} x_{0}$ are all in $V$. This argument shows that the set $N\left(x_{0}, U\right)$ is not syndetic, contradicting our assumption that $x_{0}$ is uniformly recurrent.

The next three lemmas are well known; see e.g. [12].

4.4. Lemma. Let $(X, T)$ be a dynamical system, $U \subset X$ a nonempty open subset and $x \in X$. Then $N(U, U) \supset N(x, U)-N(x, U)$. If moreover $(X, T)$ is minimal then $N(U, U)=N(x, U)-N(x, U)$

Proof. If $T^{m} x \in U$ and $T^{n} x \in U$ then $T^{n-m} T^{m} x \in U$, so that $N(U, U) \supset$ $N(x, U)-N(x, U)$. Conversely, if $n \in N(U, U)$, there is some $y \in U$ with $T^{n} y \in U$. By minimality there is some $m \in \mathbb{Z}$ such that $T^{m} x$ is sufficiently close to $y$ to ensure that both $T^{m} x \in U$ and $T^{n} T^{m} x \in U$. Then $n=$ $(n+m)-m$ and both $n+m$ and $m$ are in $N(x, U)$.

4.5. Lemma. If $S \subset \mathbb{Z}$ is syndetic then there is a minimal system $(Y, T)$ and an open nonempty $U \subset Y$ such that $S-S \supset N(U, U)$.

Proof. Let $\Omega=\{0,1\}^{\mathbb{Z}}$ and $\sigma: \Omega \rightarrow \Omega$ be the shift transformation: $(\sigma \omega)_{n}=\omega_{n+1}$. Set $Y^{\prime}=\overline{\mathcal{O}}_{\sigma}\left(\mathbf{1}_{S}\right)$ and $U^{\prime}=\left\{\omega \in \Omega: \omega_{0}=1\right\}$. It is not hard to check that $Y^{\prime}$ contains a minimal subset $Y \subset Y^{\prime}$ such that $U=Y \cap U^{\prime}$ is not empty. If $n \in N(U, U)=\left\{n: \sigma^{n} U \cap U \neq \emptyset\right\}$ then there is a point $y_{0} \in U$ with $\sigma^{n} y_{0} \in U$. There exists an $m \in \mathbb{Z}$ such that $\sigma^{m} \mathbf{1}_{S}$ is sufficiently close to $y_{0}$ to ensure that both $\sigma^{m} \mathbf{1}_{S} \in U^{\prime}$ and $\sigma^{n} \sigma^{m} \mathbf{1}_{S} \in U^{\prime}$. Thus both $m$ and $n+m$ are in $S$ and $n=n+m-m$ is in $S-S$.

The next lemma follows easily from the characterization of Kronecker systems given in Theorem 1.1; we leave the details to the reader.

4.6. Lemma. Let $(X, T)$ be a Kronecker system and $V \subset X$ a nonempty open subset. Then for every point $x_{0} \in V$ there exists an open neighborhood $x_{0} \in V_{0} \subset V$ such that

$$
N(V, V) \supset N\left(x_{0}, V\right) \supset N\left(V_{0}, V_{0}\right) .
$$

Thus, denoting by $\mathcal{E}_{\mathrm{Bo}}^{\prime}$ the collection of subsets of the form $N(x, V)$, where $(X, T)$ is Kronecker, $x \in X$ and $V$ is an open neighborhood of $x$, we have $\mathcal{F}\left(\mathcal{E}_{\mathrm{Bo}}\right)=\mathcal{F}\left(\mathcal{E}_{\mathrm{Bo}}^{\prime}\right)$, hence $\mathbf{B o}=\mathcal{F}\left(\mathcal{E}_{\mathrm{Bo}}\right)^{*}=\mathcal{E}_{\mathrm{Bo}}^{*}=\mathcal{E}_{\text {Bo }}^{\prime *}$

4.7. Definition. Let $\alpha=\left(\alpha_{1}, \ldots, \alpha_{k}\right)$, a finite sequence of real numbers, and $\epsilon>0$ be given. Set

$$
B\left(\alpha_{1}, \ldots, \alpha_{k} ; \epsilon\right)=\{n \in \mathbb{Z}:\|n \alpha\|<\epsilon\} .
$$


Here $\alpha$ is considered as an element of the $k$-torus, $\mathbb{T}^{k}=(\mathbb{R} / \mathbb{Z})^{k}$, and for $x \in$ $\mathbb{R}^{k},\|x\|$ denotes the Euclidian distance of $x$ from $\mathbb{Z}^{k}$. We say that a subset $B$ of $\mathbb{Z}$ is a Bohr neighborhood of zero if it contains some $B\left(\alpha_{1}, \ldots, \alpha_{k} ; \epsilon\right)$.

Since by Kronecker's theorem the equicontinuous dynamical system $\left(\mathbb{T}^{k}, T\right)$, where $T x=x+\alpha(\bmod 1)$ with $\left\{1, \alpha_{1}, \ldots, \alpha_{k}\right\}$ independent over the rational numbers, is a minimal system, it follows that every Bohr neighborhood of zero is in $\mathcal{F}\left(\mathcal{E}_{\mathrm{Bo}}\right)$. (Take $V=B_{\epsilon}(0) \subset \mathbb{T}^{k}$, so that $B\left(\alpha_{1}, \ldots, \alpha_{k} ; \epsilon\right.$ ) $=N(0, V)$.) Since for every Kronecker system, factors of the form $\left(\mathbb{T}^{k}, R_{\alpha}\right)$ and finite cyclic permutations separate points, this leads to the following characterizations of Bohr neighborhoods of zero. (For more details see e.g. [13].)

4.8. Proposition. The following conditions on a subset $B \subset \mathbb{Z}$ are equivalent:

(1) $B$ is a Bohr neighborhood of zero.

(2) $B$ is in $\mathcal{F}\left(\mathcal{E}_{\mathrm{Bo}}\right)$; that is, $B$ contains a subset of the form $N(V, V)$ where $(X, T)$ is a Kronecker system and $V$ a nonempty open subset of $X$.

(3) $\operatorname{cls} \phi(B)$ is a neighborhood of the zero element in the compact mono-

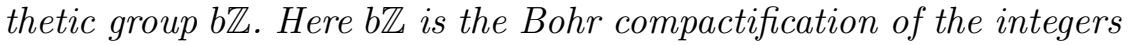
and $\phi: \mathbb{Z} \rightarrow b \mathbb{Z}$ is the natural embedding.

4.9. Problem $\left(\mathrm{A}^{\prime}\right)$. Given a syndetic subset $S \subset \mathbb{Z}$, is $S-S$ a Bohr neighborhood of zero? That is, is there a set $B=B\left(\alpha_{1}, \ldots, \alpha_{k} ; \epsilon\right)$ with $S-S \supset B ?$

The following assertion is well known but we provide a proof for completeness.

Claim. Problem $\left(\mathrm{A}^{\prime}\right)$ is a reformulation of Problem (A).

Proof. To see this assume first that the answer to Problem $\left(A^{\prime}\right)$ is in the affirmative. Let $(X, T)$ be a minimal system and $U \subset X$ a nonempty open subset. Then, by Lemma $4.4, N(U, U)=S-S$, where $S=N\left(x_{0}, U\right)$ for some (any) $x_{0} \in X$. By Lemma $4.3, S$ is syndetic and by our assumption $S-S$ and therefore also $N(U, U)$ contain a Bohr neighborhood of zero. By Proposition 4.8 we conclude that every $N(U, U)$, i.e. every member of $\mathcal{E}_{\text {Bir }}$, contains a member of $\mathcal{E}_{\text {Bo }}$, whence $\mathcal{F}\left(\mathcal{E}_{\text {Bir }}\right)=\mathcal{F}\left(\mathcal{E}_{\text {Bo }}\right)$, and Bir $=$ Bo (see Remark 2.6 above).

Conversely, assume now that $\mathbf{B i r}=\mathbf{B o}$, and let $S \subset \mathbb{Z}$ be a syndetic subset. By Lemma 4.5, $S-S$ contains a set of the form $N(U, U)$ for some minimal system $(X, T)$ and an open nonempty $U \subset X$. If the set $S-S$ is not a Bohr neighborhood of zero then, for every Kronecker system $(Y, T)$ and nonempty open $V \subset Y, N(V, V) \cap N(U, U)^{\mathrm{c}} \neq \emptyset$, and therefore $N(U, U)^{\mathrm{c}}$ 
is in Bo. This contradicts our assumption since $N(U, U) \cap N(U, U)^{\mathrm{c}}=\emptyset$ implies that $N(U, U)^{\mathrm{c}}$ is not in Bir.

We do have the following facts:

4.10. Theorem. Let $S \subset \mathbb{Z}$ be a syndetic subset.

(1) (Veech [27]) There exists a Bohr neighborhood of zero B such that $(S-S) \triangle B$ is a subset of upper Banach density zero.

(2) (Ellis and Keynes [7]) There exists a Bohr neighborhood of zero B with $S-S+S-s \supset B$ for some $s \in S$.

Recall that a topological group $G$ is called minimally almost periodic (MAP) if it admits no nontrivial continuous homomorphism into a compact group. Or, equivalently, if it admits no nontrivial minimal equicontinuous action on a compact space. There are many examples of MAP monothetic Polish groups (see e.g. [1]). A topological group $G$ has the fixed point on compacta property (FPC) if every compact $G$-dynamical system has a fixed point; see [18] and [12]. Some authors call this property extreme amenability. Recently, the theory of Polish groups with the fixed point on compacta property received a lot of attention and new and exciting connections with other branches of mathematics (like Ramsey theory, Gromov's theory of mm-spaces, and concentration of measure phenomena) were discovered; see V. Pestov's book [24]. In [12] it is shown that the Polish group $G$ of all measurable functions $f$ from a nonatomic Lebesgue measure space $(\Omega, \mathcal{B}, m)$ into the circle $\{z \in \mathbb{C}:|z|=1\}$, with pointwise product and the topology of convergence in measure, is monothetic and has the FPC property. Of course, every topological group with the FPC property is also MAP. The following problem is posed in [12].

4.11. Problem $\left(\mathrm{A}^{\prime \prime}\right)$. Is there a Polish monothetic group which is MAP but does not have the fixed point on compacta property?

It is shown there that a positive answer to Problem $\left(\mathrm{A}^{\prime \prime}\right)$ would provide a negative answer to Problem $\left(\mathrm{A}^{\prime}\right)$.

5. More on topological recurrence. Let $(X, T)$ be a dynamical system, where $X$ is a compact metrizable space and $T: X \rightarrow X$ is a homeomorphism of $X$ onto itself. We fix a compatible metric $d$ on $X$. Recall the following familiar definition. A point $x \in X$ is recurrent if for every $\epsilon>0$ there is an $n \in \mathbb{Z} \backslash\{0\}$ with $d\left(T^{n} x, x\right)<\epsilon$. Equivalently, setting

$$
\phi(x)=\inf \left\{d\left(T^{n} x, x\right): n \in \mathbb{Z} \backslash\{0\}\right\},
$$

we see that $x$ is recurrent iff $\phi(x)=0$. More generally, given an infinite subset $L \subset \mathbb{Z} \backslash\{0\}$, set

$$
\phi_{L}(x)=\inf \left\{d\left(T^{n} x, x\right): n \in L\right\},
$$


and call a point $x \in X, L$-recurrent when $\phi_{L}(x)=0$. Let us remark that the role of the metric $d$ in these definitions is not essential. It is not hard to show that although the functions $\phi_{L}$ usually depend on the choice of a compatible metric $d$, the sets of $L$-recurrent points do not. We say that a subset $A \subset X$ is wandering if there is an infinite set $J \subset \mathbb{Z}$ such that the sets $T^{j} A, j \in J$, are pairwise disjoint. We say that the system $(X, T)$ is nonwandering if $X$ contains no nonempty wandering open subsets. Following Furstenberg, $[10$, Theorem 1.27], we have:

\subsection{Theorem.}

(1) The function $\phi_{L}$ is upper-semicontinuous.

(2) The set of L-recurrent points is a $G_{\delta}$ subset of $X$.

(3) If $(X, T)$ is nonwandering then the set of recurrent points is a dense $G_{\delta}$ subset of $X$.

(4) If there is a $T$-invariant probability measure $\mu$ on $X$ with full support (i.e. $\mu(U)>0$ for every nonempty open $U$ ) and $L$ is a Poincaré set then the set of $L$-recurrent points is a dense $G_{\delta}$ subset of $X$.

(5) If $(X, T)$ is minimal and $L$ is a Birkhoff set then the set of $L$ recurrent points is a dense $G_{\delta}$ subset of $X$.

Proof. We leave the proofs of claims (1) and (2) as an exercise. For (3) see Furstenberg [10, Theorem 1.27] (or adapt the following proof). For the proof of claim (4) we first recall that an upper-semicontinuous function on $X$ has a dense $G_{\delta}$ set of continuity points. Let $X_{L} \subset X$ be the dense $G_{\delta}$ set of continuity points of $\phi_{L}$. Suppose $\phi_{L}\left(x_{0}\right)=a>0$ for some $x_{0} \in X_{L}$. Then, by continuity, there is a $0<\delta<a / 4$ such that $\phi_{L}(x)>a / 2$ for every $x$ is an open ball $U$ of radius $\delta$ around $x_{0}$. Since $\mu(U)>0$ and $L$ is Poincaré we have $L \cap N(U, U) \neq \emptyset$. For $n$ in this intersection there are $u_{1}, u_{2} \in U$ with $T^{n} u_{1}=u_{2}$, hence $d\left(u_{1}, T^{n} u_{1}\right)<a / 2$. In particular, $\phi_{L}\left(u_{1}\right)<a / 2$. This contradicts our choice of $U$ and we conclude that $\phi_{L}(x)=0$ for every $x \in X_{L}$. This completes the proof of claim (4). A similar argument will prove claim (5).

In the next two theorems we establish several characterizations of Birkhoff sets. We will use the following lemma which is valid for every minimal system.

5.2. Lemma. Let $(X, T)$ be a minimal system and let $\eta>0$ be given. Then there exists a positive integer $M \geq 1$ such that for every $x \in X$ the set $\left\{T^{j} x\right\}_{j=0}^{M}$ is $\eta$-dense in $X$; that is, for every $x^{\prime} \in X$ there is some $0 \leq j \leq M$ with $d\left(x^{\prime}, T^{j} x\right)<\eta$.

Proof. Assuming the contrary we would have, for each $n$, points $x_{n}, y_{n} \in X$ such that $B_{\eta}\left(y_{n}\right) \cap\left\{T^{j} x_{n}\right\}_{j=0}^{n}=\emptyset$. By compactness there are convergent 
subsequences, say $x_{n_{j}} \rightarrow x$ and $y_{n_{j}} \rightarrow y$. By minimality there is a positive $m \geq 1$ such that $d\left(T^{m} x, y\right)<\eta / 3$. We now choose $j$ so large that: (i) $n_{j}>m$, (ii) $d\left(y, y_{n_{j}}\right)<\eta / 3$, and (iii) $x_{n_{j}}$ is sufficiently close to $x$ to ensure that $d\left(T^{m} x_{n_{j}}, T^{m} x\right)<\eta / 3$. With this choice of $j$ we now have $d\left(T^{m} x_{n_{j}}, y_{n_{j}}\right)<d\left(T^{m} x_{n_{j}}, T^{m} x\right)+d\left(T^{m} x, y\right)+d\left(y, y_{n_{j}}\right)<\eta / 3+\eta / 3+\eta / 3=\eta$. Since $n_{j}>m$, this contradicts the choice of $x_{n_{j}}$ and $y_{n_{j}}$.

5.3. TheOREM. The following conditions on a subset $L \subset \mathbb{Z}_{*}$ are equivalent:

(1) $L$ is Birkhoff.

(2) $L \cap(S-S) \neq \emptyset$ for every syndetic subset $S \subset \mathbb{Z}$.

(3) For every minimal dynamical system $(X, T)$, the set of $L$-recurrent points is dense and $G_{\delta}$.

(4) For every dynamical system $(X, T)$ and $\epsilon>0$ there are $x \in X$ and $m \in L$ with $d\left(T^{m} x, x\right)<\epsilon$.

Proof. From Lemmas 4.4 and 4.5 we easily deduce the equivalence of properties (1) and (2). The implication $(1) \Rightarrow(3)$ is proven in Theorem 5.1.5. Next assume (3). Given a minimal system $(X, T)$ and a nonempty open subset $U \subset X$ we clearly have $L \cap N(U, U) \neq \emptyset$, whence $L$ is Birkhoff. Thus $(3) \Rightarrow(1)$.

As every dynamical system has a minimal subsystem we clearly have $(3) \Rightarrow(4)$. Finally, we show that (4) implies $(3)$. Let $(X, T)$ be a minimal system. For $\epsilon>0$ set

$$
V_{L}(\epsilon)=\left\{x \in X: \exists m \in L \text { with } d\left(T^{m} x, x\right)<\epsilon\right\} .
$$

Clearly, $V_{L}(\epsilon)$ is open and assuming (4) we know that it is nonempty. Given $\eta>0$ there is, by Lemma 5.2 , an $M \geq 1$ such that for every $x \in X$ the set $\left\{T^{i} x\right\}_{i=0}^{M}$ is $\eta$-dense. Let $\delta>0$ be such that $d\left(x, x^{\prime}\right)<\delta$ implies $d\left(T^{i} x, T^{i} x^{\prime}\right)<\epsilon$ for every $0 \leq i \leq M$. It now follows that for every $x \in V_{L}(\delta)$, we have $\left\{T^{i} x\right\}_{i=0}^{M} \subset V_{L}(\epsilon)$, and consequently, that $V_{L}(\epsilon)$ is $\eta$-dense. Since $\eta$ is arbitrary, we conclude that $V_{L}(\epsilon)$ is dense. By Baire's theorem we conclude that $X_{0}=\bigcap_{\epsilon>0} V_{L}(\epsilon)$ is a dense $G_{\delta}$ subset of $X$. Clearly, every $x \in X_{0}$ is $L$-recurrent.

In order to achieve additional characterizations for Birkhoff sets we introduce the following definition. For $r \in \mathbb{N}$ we define $\mathbb{N}_{r}=\{1, \ldots, r\}$.

5.4. Definition. Let $r \in \mathbb{N}$. A subset $L \subset \mathbb{Z}_{*}$ is said to be $r$-Birkhoff (notation: $L \in \mathbf{B i r}_{r}$ ) if the following two equivalent conditions hold:

(1) For every sequence $\left\{z_{i}\right\}_{i \in \mathbb{Z}}$ over $\mathbb{N}_{r}$, there are $m \in L$ and $i \in \mathbb{Z}$ such that $z_{i}=z_{i+m}$.

(2) For every coloring $c: \mathbb{Z} \rightarrow \mathbb{N}_{r}$ there are $i, j \in \mathbb{Z}$ with $c(i)=c(j)$ and $i-j \in L$. 
5.5. Remark. In the above definition one can replace $\mathbb{Z}$ by $\mathbb{N}$.

5.6. TheOREM. The following conditions on a subset $L \subset \mathbb{Z}_{*}$ are equivalent:

(1) L is Birkhoff.

(2) For any compact metric space $(Z, d)$, every sequence $\left\{z_{i}\right\}_{i \in \mathbb{Z}}$ with $z_{i} \in Z$, and every $\epsilon>0$, there are $m \in L$ and $i \in \mathbb{Z}$ such that $d\left(z_{i}, z_{i+m}\right)<\epsilon$.

(3) $L$ is $r$-Birkhoff for all $r \in \mathbb{N}$.

Thus, by the above theorem, $\mathbf{B i r}=\bigcap_{r \in \mathbb{N}} \mathbf{B i r}_{r}$.

Proof. (1) $\Rightarrow(2)$ : Suppose $L$ is Birkhoff and let $Y=\overline{\mathcal{O}}_{T}(\zeta)$, where $\Omega=$ $Z^{\mathbb{Z}}, T: \Omega \rightarrow \Omega$ is the shift and the element $\zeta \in \Omega$ is defined by $\zeta(i)=z_{i}$. Let $M \subset Y$ be a minimal subset. Applying Theorem 5.3 we see that there is a point $x \in M \subset Y$ which is $L$-recurrent. Fix a compatible metric $\rho$ on $\Omega$ and let $0<\delta$ be such that $\rho\left(\omega, \omega^{\prime}\right)<\delta$ implies $d\left(\omega(0), \omega^{\prime}(0)\right)<\epsilon$. Let $m \in L$ be such that $d\left(T^{m} x, x\right)<\delta$. Let $i \in \mathbb{Z}$ be chosen so that $T^{i} \zeta$ is sufficiently close to $x$ to ensure that also $\rho\left(T^{m} T^{i} \zeta, T^{i} \zeta\right)<\delta$. By our choice of $\delta$ we have $d\left(z_{m+i}, z_{i}\right)=d(\zeta(m+i), \zeta(i))<\epsilon$.

$(2) \Rightarrow(3)$ : Take $Z=\mathbb{N}_{r}=\{1, \ldots, r\}$. Let $d(i, j)=\delta_{i j}$ for $i, j \in \mathbb{N}_{r}$ and take $\epsilon=1 / 2$. Then $d\left(z_{i}, z_{i+m}\right)<\epsilon$ implies $z_{i}=z_{i+m}$.

$(3) \Rightarrow(1)$ : We will show that condition (4) in Theorem 5.3 is satisfied. So let a minimal system $(X, T)$ and $\epsilon>0$ be given. Let $\left\{V_{i}\right\}_{i=1}^{r}$ be an open cover of $X$ by balls of radius $\epsilon / 2$. Fix $x_{0} \in X$ and choose a sequence $z_{i} \in \mathbb{N}_{r}$ such that $T^{i} x_{0} \in U_{z_{i}}$ for every $i \in \mathbb{Z}$. By (3) we have $m \in L$ and $i \in \mathbb{Z}$ such that $z_{m+i}=z_{i}=j$, whence $T^{i} x_{0}$ and $T^{m+i} x_{0}$ are both in $U_{j}$. Thus $d\left(T^{m} T^{i} x_{0}, T^{i} x_{0}\right)<\epsilon$, and taking $x=T^{i} x_{0}$ we have the required $x$.

5.7. Remark. The last condition in Theorem 5.6 can be formulated as a coloring property: For every $r$ and every coloring $c: \mathbb{Z} \rightarrow\{1, \ldots, r\}$ there are $i, j \in \mathbb{Z}$ with $c(i)=c(j)$ and $i-j \in L$. See [28] for a graph-theoretical interpretation of this coloring property.

We will now consider some basic properties of $r$-Birkhoff sets. We leave the first statement as an easy exercise.

5.8. Proposition (A compactness principle). For $r \geq 1$, every $r$-Birkhoff set contains a finite r-Birkhoff subset.

For any $r \in \mathbb{N}$, each of the sets $k \mathbb{N}_{r}=\{k, 2 k, \ldots, r k\}, k \in \mathbb{N}$, is $r$-Birkhoff. Indeed, let $\left(z_{i}\right)$ be an arbitrary sequence in $\mathbb{N}_{r}$. Since card $\left(k \mathbb{N}_{r+1}\right)$ $=r+1>r=\operatorname{card}\left(\mathbb{N}_{r}\right)$, there are $i, j \in k \mathbb{N}_{r+1}, i \neq j$, such that $z_{i}=z_{j}$. Assuming, with no loss of generality, that $m=j-i>0$, we get $z_{i}=z_{i+m}$ with some $m \in k \mathbb{N}_{r}$, completing the proof (see Definition 5.4(1)). 
On the other hand, for finite subsets $M \subset \mathbb{Z}_{*}$ the following implication holds:

$$
\operatorname{card}(M)=r \geq 1 \Rightarrow M \notin \mathbf{B i r}_{r+1} .
$$

Indeed, with no loss of generality we may assume that $M \subset \mathbb{N}$ (by replacing $M$ with the set $(M \cup(-M)) \cap \mathbb{N})$. Construct a sequence $\left\{z_{i}\right\}_{i \in \mathbb{Z}}$ over the set $\mathbb{N}_{r+1}=\{1, \ldots, r+1\}$ as follows. For $i \leq 0$, set $z_{i}=1$; for $i \geq 1$, set inductively

$z_{i}=\min X_{i}, \quad$ where $\quad X_{i}=\left\{x \in \mathbb{N}_{r+1}: x \neq z_{i-m}\right.$ for all $\left.m \in M\right\}$.

(Clearly, $X_{i} \neq \emptyset$ for $i \geq 1$, because $\operatorname{card}(M)=r<r+1=\operatorname{card}\left(\mathbb{N}_{r+1}\right)$.) The above construction implies that $z_{i}=z_{i+m}$ has no solutions in $i \in \mathbb{N}$ and $m \in M$. It follows that $M \notin \mathbf{B i r}_{r+1}$ (see Definition 5.4 and the subsequent remark).

We conclude that the sets $k \mathbb{N}_{r}=\{k, 2 k, \ldots, r k\}, k, r \in \mathbb{N}$, provide examples of $r$-Birkhoff sets which are not $(r+1)$-Birkhoff. More refined examples will be provided next (see (5.1) below).

5.9. Definition. A subset $M \subset Z_{*}$ is called stably $r$-Birkhoff (notation: $\left.M \in \mathbf{B i r}_{r}^{\prime}\right)$ if for every finite subset $F \subset \mathbb{Z}$, the difference set $M \backslash F$ is $r$-Birkhoff.

Define the sets

$$
L_{r}=\left\{n(r+2)^{k}: n \in\{1, \ldots, r\}, k \geq 0\right\} \subset \mathbb{N} \quad \text { for } r \in \mathbb{N} .
$$

We claim that, for every $r \geq 2$, the set $L_{r}$ is:

(a) lacunary;

(b) stably $r$-Birkhoff;

(c) not $(r+1)$-Birkhoff.

The fact that $L_{r}$ is lacunary is clear. In fact, if $\left\{x_{1}<x_{2}<\cdots\right\}$ is the linear ordering of $L_{r}$, then $\min _{k \geq 1} x_{k+1} / x_{k}=r /(r-1)$ for $r \geq 2$.

The set $L_{r}$ is stably $r$-Birkhoff because $L_{r}$ can be represented as a disjoint infinite union $L_{r}=\bigcup_{k \geq 0} L_{r, k}$ where each $L_{r, k}=(r+2)^{k} \mathbb{N}_{r}$ is $r$-Birkhoff (as proved earlier).

Finally, to prove that $L_{r}$ is not $(r+1)$-Birkhoff, define a sequence $\left\{z_{k}\right\}_{k \in \mathbb{Z}}$ over $\mathbb{N}_{r+1}$ by the condition $z_{i} \equiv i(\bmod r+1)$. We claim that $z_{i}=z_{i+m}$ has no solution in $m \in L_{r}$ and $i \in \mathbb{Z}$. Indeed, otherwise

$$
i \equiv i+m(\bmod r+1) \Rightarrow m \equiv 0(\bmod r+1) \Rightarrow \frac{m}{r+1} \in \mathbb{Z},
$$

which is impossible (see (5.1)). This completes the proof that $L_{r} \notin \mathbf{B i r}_{r+1}$ (see Definition 5.4(1)).

The fact that the sets $L_{r} \in \mathbf{B i r}_{r}^{\prime}$ are lacunary should be compared with the fact that no set in Bo (which by Lemma 2.4 contains Bir) is lacunary (see the examples in Section 3). 
6. The moving recurrence problem. The following question was recently posed by Boshernitzan, and is still open.

6.1. Problem (B). Let $(X, T)$ be a dynamical system, $\mu \in M_{T}(X)$ a $T$-invariant probability measure on $X$ and $\left(n_{k}\right)$ an infinite sequence of integers. Define

$$
\psi_{\left(n_{k}\right)}(x)=\inf _{k \geq 1} d\left(T^{n_{k}} x, T^{n_{k}+k} x\right) .
$$

Is it true that $\psi_{\left(n_{k}\right)}(x)=0, \mu$-a.e.?

In this section we prove a topological analogue using the tools developed in the previous sections.

6.2. Definition. For a sequence $\left(n_{k}\right)$ of elements of $\mathbb{Z}$, let

$$
\psi_{\left(n_{k}\right)}(x)=\inf _{k \geq 1} d\left(T^{n_{k}+k} x, T^{n_{k}} x\right) .
$$

More generally, given two sequences $\left(n_{k}\right)$ and $\left(r_{k}\right)$ of elements of $\mathbb{Z}$, let

$$
\psi_{\left(n_{k}, r_{k}\right)}(x)=\inf _{k \geq 1} d\left(T^{n_{k}+r_{k}} x, T^{n_{k}} x\right) .
$$

We say that a point $x \in X$ is $\left(n_{k}\right)$-moving recurrent if $\psi_{\left(n_{k}\right)}(x)=0$. It is $\left(n_{k}, r_{k}\right)$-moving recurrent when $\psi_{\left(n_{k}, r_{k}\right)}(x)=0$. Note that $\psi_{\left(n_{k}\right)}=\psi_{\left(n_{k}, k\right)}$.

Again we have:

6.3. LEMMA. The function $\psi_{\left(n_{k}, r_{k}\right)}$ is upper-semicontinuous and the set of $\left(n_{k}, r_{k}\right)$-moving recurrent points is a $G_{\delta}$ subset of $X$.

6.4. TheOREM. Let $\left(r_{k}\right)$ be a Birkhoff set. Then for every sequence $\left(n_{k}\right)$ and every minimal dynamical system $(X, T)$, the set of $\left(n_{k}, r_{k}\right)$-moving recurrent points is dense and $G_{\delta}$. In particular, taking $r_{k}=k$ we see that for every minimal dynamical system $(X, T)$ the set of $\left(n_{k}\right)$-moving recurrent points is dense and $G_{\delta}$.

Proof.

SteP 1. Let $X_{0} \subset X$ denote the dense $G_{\delta}$ set of continuity points of $\psi_{\left(n_{k}, r_{k}\right)}$ (Lemma 6.3). Let $x_{0} \in X_{0}$ and assume that $\psi_{\left(n_{k}, r_{k}\right)}\left(x_{0}\right)=2 \epsilon>0$. Since $x_{0}$ is a continuity point we can find a ball $U$ around $x_{0}$ such that $\psi_{\left(n_{k}, r_{k}\right)}(x)>\epsilon$ for every $x \in U$.

STEP 2. We will show that the set

$$
V(\epsilon)=\left\{x \in X: \psi_{\left(n_{k}, r_{k}\right)}(x)<\epsilon\right\}
$$

is dense.

Fix $\eta>0$ and use Lemma 5.2 to find $M \geq 1$ such that for every $x \in X$ the set $\left\{T^{j} x\right\}_{j=0}^{M}$ is $\eta$-dense in $X$. Then choose $\delta>0$ such that $d\left(x, x^{\prime}\right)<\delta$ implies $d\left(T^{j} x, T^{j} x^{\prime}\right)<\epsilon$ for every $0 \leq j \leq M$. 
Next observe that there exist $x \in X$ and $k \geq 1$ with $d\left(T^{n_{k}+r_{k}} x, T^{n_{k}} x\right)<\delta$. In fact, since $\left(r_{k}\right)$ is Birkhoff, we can (applying Theorem 5.3) pick an $\left(r_{k}\right)$ recurrent point $x^{\prime} \in X$ and then find $k \geq 1$ with $d\left(T^{r_{k}} x^{\prime}, x^{\prime}\right)<\delta$. Set $x=T^{-n_{k}} x^{\prime}$, so that $x^{\prime}=T^{n_{k}} x$ and

$$
d\left(T^{n_{k}+r_{k}} x, T^{n_{k}} x\right)=d\left(T^{r_{k}} x^{\prime}, x^{\prime}\right)<\delta .
$$

Now, by the choice of $\delta$, we have

$$
d\left(T^{n_{k}+r_{k}} T^{j} x, T^{n_{k}} T^{j} x\right)<\epsilon \quad \text { for all } 0 \leq j \leq M .
$$

Thus $\psi_{\left(n_{k}, r_{k}\right)}\left(T^{j} x\right)<\epsilon$ for all $0 \leq j \leq M$ and we conclude that $V(\epsilon)$ is $\eta$-dense. As this holds for every $\eta>0$ we conclude that $V(\epsilon)$ is dense.

SteP 3. Now with $U$ as in Step 1 we have $U \cap V(\epsilon) \neq \emptyset$ and we reached the contradiction $\epsilon<\psi_{\left(n_{k}, r_{k}\right)}(x)<\epsilon$ for any point $x$ in this intersection. Since the assumption $\psi_{\left(n_{k}, r_{k}\right)}\left(x_{0}\right)>0$ leads to a contradiction we conclude that $\psi_{\left(n_{k}, r_{k}\right)}\left(x_{0}\right)=0$ for every $x_{0} \in X_{0}$, as required.

Recall the following definition from [16].

6.5. Definition. A dynamical system $(X, T)$ is called an $M$-system if (i) it is topologically transitive, and (ii) the union of the minimal subsystems of $X$ is dense in $X$.

The class of $M$-systems is very large, e.g. it contains every infinite topologically transitive system with a dense set of periodic points. (The latter systems are called chaotic in the sense of Devaney, or P-systems.)

6.6. Corollary. Let $(X, T)$ be an $M$-system and $\left(n_{k}\right)$ an infinite sequence in $\mathbb{Z}$. Then there is a dense $G_{\delta}$ subset $X_{0} \subset X$ such that $\psi_{\left(n_{k}\right)}(x)=0$ for every $x \in X_{0}$. In particular, the set $X_{\operatorname{tr}} \cap X_{0}$ of $\left(n_{k}\right)$-moving recurrent transitive points is a dense $G_{\delta}$ subset of $X$.

Proof. The set $X_{0}=\left\{x \in X: \psi_{\left(n_{k}\right)}(x)=0\right\}$ is a $G_{\delta}$ subset of $X$. By Theorem 6.4 for every minimal subset $M \subset X$ the set $M_{0}=M \cap X_{0}$ is a dense $G_{\delta}$ subset of $M$. Thus $\bigcup\left\{M_{0}: M\right.$ is a minimal subset of $\left.X\right\} \subset$ $X_{0}$ is dense in $\bigcup\{M: M$ is a minimal subset of $X\}$. In turn, the latter is dense in $X$ and it follows that $X_{0}$ is dense in $X$. Finally, as the set $X_{\operatorname{tr}}$ of transitive points in an $M$-system is always dense and $G_{\delta}$ we conclude that so is $X_{\operatorname{tr}} \cap X_{0}$.

\section{Absolute moving recurrence}

7.1. Definition. We will say that a system $(X, T)$ is absolutely moving recurrent if for every infinite sequence $\left(n_{k}\right) \subset \mathbb{Z}$ we have $\psi_{\left(n_{k}\right)} \equiv 0$ (i.e. every point of $X$ is $\left(n_{k}\right)$-moving recurrent).

Recall the following definition from [15]: 
7.2. Definition. A dynamical system $(X, T)$ is called uniformly rigid if there exists a sequence $m_{i} \nearrow \infty$ in $\mathbb{Z}$ such that

$$
\lim _{i \rightarrow \infty} \sup _{x \in X} d\left(x, T^{m_{i}} x\right)=0 .
$$

For any dynamical system $(X, T)$ let

$$
\Lambda(X, T)=\text { unif-cls }\left\{T^{n}: n \in \mathbb{Z}\right\} \subset \operatorname{Homeo}(X)
$$

be the uniform closure of the powers of $T$ in the Polish group Homeo $(X)$. Of course, $\Lambda(X, T)$ is a Polish monothetic group, and the system $(X, T)$ is uniformly rigid iff $\Lambda(X, T)$ is not discrete.

7.3. Theorem. A topologically transitive dynamical system $(X, T)$ is absolutely moving recurrent if and only if it is uniformly rigid.

Proof. Suppose first that $(X, T)$ is uniformly rigid with $T^{m_{i}} \stackrel{\text { unif }}{\longrightarrow}$ Id. Let $\left(n_{k}\right) \subset \mathbb{Z}$ be an arbitrary infinite sequence. Then for every $\epsilon>0$ there exists an $i_{0}$ such that for $i>i_{0}, d\left(T^{m_{i}} x, x\right)<\epsilon$ for every $x \in X$. In particular then,

$$
d\left(T^{n_{m_{i}}+m_{i}} x, T^{n_{m_{i}}} x\right)=d\left(T^{m_{i}}\left(T^{n_{m_{i}}} x\right), T^{n_{m_{i}}} x\right)<\epsilon .
$$

Thus, $\liminf _{k} d\left(T^{n_{k}+k} x, T^{n_{k}} x\right)=0$ for every $x \in X$, and we have shown that $(X, T)$ is absolutely moving recurrent. (Note that topological transitivity is not needed in this direction.)

Conversely, suppose $(X, T)$ is not uniformly rigid, that is,

$$
\exists \epsilon_{0}>0 \forall k \geq 1 \exists x_{k} \in X \quad d\left(T^{k} x_{k}, x_{k}\right)>\epsilon_{0} .
$$

Fix $x_{0} \in X_{\text {tr }}$ and for each $k$ choose $n_{k} \in \mathbb{N}$ such that $T^{n_{k}} x_{0}$ is sufficiently close to $x_{k}$ to ensure that also $d\left(T^{n_{k}+k} x_{0}, T^{n_{k}} x_{0}\right)=d\left(T^{k} T^{n_{k}} x_{0}, T^{n_{k}} x_{0}\right)$ $>\epsilon_{0}$. For the sequence $\left(n_{k}\right)$ we have $\psi_{\left(n_{k}\right)}\left(x_{0}\right) \geq \epsilon_{0}$, hence $(X, T)$ is not absolutely moving recurrent.

Acknowledgements. This work was done while both authors participated in the program "Ergodic Theory and Additive Combinatorics" at MSRI in the summer of 2008. We thank MSRI for its support. We also thank Benjy Weiss for very helpful discussions.

The second author's work is supported in part by ISF grant \#1157/08.

\section{References}

[1] M. Ajtai, I. Havas and J. Komlós, Every group admits a bad topology, in: Studies in Pure Math., P. Erdős (ed.), Birkhäuser, Basel, 1983, 21-34.

[2] E. Akin, Recurrence in Topological Dynamics. Furstenberg Families and Ellis Actions, Plenum Press, New York, 1997.

[3] E. Akin and E. Glasner, Residual properties and almost equicontinuity, J. Anal. Math. 84 (2001), 243-286. 
[4] V. Bergelson, H. Furstenberg and B. Weiss, Piecewise-Bohr sets of integers and combinatorial number theory, in: Topics in Discrete Mathematics. Dedicated to Jarik Nešetřil on the Occasion of His 60th Birthday, M. Klazar et al. (eds.), Algorithms Combin. 26, Springer, Berlin, 2006, 13-37.

[5] M. Boshernitzan, Homogeneously distributed sequences and Poincaré sequences of integers, Monatsh. Math. 96 (1983), 173-181.

[6] J. Bourgain, On the maximal ergodic theorem for certain subsets of the integers, Israel J. Math. 61 (1988), 39-72.

[7] R. Ellis and H. Keynes, Bohr compactifications and a result of Følner, Israel J. Math. 12 (1972), 314-330.

[8] P. Erdős, Repartition Modulo 1, Lecture Notes in Math. 475, Springer, New York, 1975 .

[9] N. Frantzikinakis and R. McCutcheon, Ergodic Theory: Recurrence, Encyclopedia of Complexity and System Science, to appear; arXiv:0705.0033 (math.DS).

[10] H. Furstenberg, Recurrence in Ergodic Theory and Combinatorial Number Theory, Princeton Univ. Press, Princeton, NJ, 1981.

[11] E. Glasner, Divisible properties and the Stone-Čech compactification, Canad. J. Math. 32 (1980), 993-1007.

[12] —, On minimal actions of Polish groups, Topology Appl. 85 (1998), 119-125.

[13] —, Ergodic Theory via Joinings, Math. Surveys Monogr. 101, Amer. Math. Soc., Providence, RI, 2003.

[14] -, Classifying dynamical systems by their recurrence properties, Topol. Methods Nonlinear Anal. 24 (2004), 21-40.

[15] E. Glasner and D. Maon, Rigidity in topological dynamics, Ergodic Theory Dynam. Systems 9 (1989), 309-320.

[16] E. Glasner and B. Weiss, Sensitive dependence on initial conditions, Nonlinearity 6 (1993), 1067-1075.

[17] W. H. Gottschalk and G. A. Hedlund, Topological Dynamics, Amer. Math. Soc. Colloq. Publ. 36, Amer. Math. Soc., Providence, RI, 1955.

[18] M. Gromov and V. D. Milman, A topological application of the isoperimetric inequality, Amer. J. Math. 105 (1983), 843-854.

[19] W. Huang, and X. Ye, An explicit scattering, non-weakly mixing example and weak disjointness, Nonlinearity 15 (2002), 849-862.

[20] Y. Katznelson, Chromatic numbers of Cayley graphs on $\mathbb{Z}$ and recurrence, in: Paul Erdős and His Mathematics (Budapest, 1999), Combinatorica 21 (2001), 211-219.

[21] I. Kříž, Large independent sets in shift-invariant graphs. Solution of Bergelson's problem, Graphs Combin. 3 (1987), 145-158.

[22] B. de Mathan, Numbers contravening a condition in density modulo 1, Acta Math. Acad. Sci. Hungar. 36 (1980), 237-241.

[23] R. McCutcheon, Three results in recurrence, in: Ergodic Theory and Its Connections with Harmonic Analysis (Alexandria, 1993), K. E. Petersen and I. A. Salama (eds.), London Math. Soc. Lecture Note Ser. 205, Cambridge Univ. Press, Cambridge, 1995, 349-358.

[24] V. Pestov, Dynamics of Infinite-Dimensional Groups. The Ramsey-DvoretzkyMilman Phenomenon, Univ. Lecture Ser. 40, Amer. Math. Soc., Providence, RI, 2006.

[25] A. D. Pollington, On the density of sequence $\left\{n_{k} \xi\right\}$, Illinois J. Math. 23 (1979), $511-515$. 
[26] I. Z. Ruzsa, Uniform distribution, positive trigonometric polynomials and difference sets, in: Seminar on Number Theory, 1981/1982, Exp. No. 18, Univ. Bordeaux I, Talence, 1982, 18 pp.

[27] W. A. Veech, The equicontinuous structure relation for minimal Abelian transformation groups, Amer. J. Math. 90 (1968), 723-732.

[28] B. Weiss, Single Orbit Dynamics, CBMS Reg. Conf. Ser. Math. 95, Amer. Math. Soc., Providence, RI, 2000.

Michael Boshernitzan

Department of Mathematics

Rice University

Houston, TX 77005, U.S.A.

E-mail: michael@rice.edu
Eli Glasner

Department of Mathematics

Tel Aviv University

Ramat Aviv, Israel

E-mail: glasner@math.tau.ac.il

Received 26 January 2009;

in revised form 24 May 2009 